\title{
M-dwarf exoplanet surface density distribution
}

\section{A log-normal fit from 0.07 to $400 \mathrm{AU}$}

\author{
Michael R. Meyer ${ }^{1,2}$, Adam Amara ${ }^{1}$, Maddalena Reggiani ${ }^{1,3}$, and Sascha P. Quanz ${ }^{1}$ \\ ${ }^{1}$ Institute for Astronomy, ETH Zurich Wolfgang-Pauli-Strasse 27, Zurich 8093, Switzerland \\ 2 Department of Astronomy, University of Michigan, Ann Arbor, MI 48109, USA \\ ${ }^{3}$ Space Sciences, Technologies and Astrophysics Research (STAR) Institute, Université de Liège, 19 allée du Six Août, 4000 Liège, \\ Belgium
}

Received 5 June 2017 / Accepted 14 July 2017

\begin{abstract}
Aims. We fit a log-normal function to the M-dwarf orbital surface density distribution of gas giant planets, over the mass range 1-10 times that of Jupiter, from 0.07 to $400 \mathrm{AU}$.

Methods. We used a Markov chain Monte Carlo approach to explore the likelihoods of various parameter values consistent with point estimates of the data given our assumed functional form.

Results. This fit is consistent with radial velocity, microlensing, and direct-imaging observations, is well-motivated from theoretical and phenomenological points of view, and predicts results of future surveys. We present probability distributions for each parameter and a maximum likelihood estimate solution.

Conclusions. We suggest that this function makes more physical sense than other widely used functions, and we explore the implications of our results on the design of future exoplanet surveys.
\end{abstract}

Key words. planets and satellites: general - stars: low-mass

\section{Introduction}

Confirmed exoplanet detections number well over a thousand, enabling statistical studies of their orbital surface density and mass distributions and of the overall frequency as a function of host star mass. Radial velocity (RV) surveys (which probe the smallest orbital separations) and direct-imaging surveys (which probe the largest separations) have been conducted for samples of M dwarfs (0.1-0.6 $M_{\odot}$; e.g., Bonfils et al. 2013; Bowler et al. 2015), FGK stars (0.5-1.5 $M_{\odot}$; e.g., Mayor et al. 2011; Chauvin et al. 2015), and A stars (1.5-2.5 $M_{\odot}$; e.g., Johnson et al. 2010; Vigan et al. 2012). A pioneering attempt to constrain both the planet mass function and orbital surface density distribution for gas giant exoplanet populations surrounding FGK stars was made by Cumming et al. (2008), who fit a homogeneous sample of RV detections, drawn from a stellar sample with well-known characteristics (Fischer \& Valenti 2005). These power-law fits revealed a rising planet mass function down to $>0.3$ times the mass of Jupiter (hereafter $M_{J}$ ) and a rising surface density distribution in units of logarithmic orbital period ( $<2000$ days). These fits have been used to plan and interpret many direct-imaging surveys representing hundreds of nights of 6-10 $\mathrm{m}$ telescope time in the past decade (e.g., Lafrenière et al. 2007; Heinze et al. 2010; Macintosh et al. 2014; Beuzit et al. 2008). Typical experiments assume a power-law surface density distribution rising with the log of the orbital radius (cf. Cumming et al. 2008) and then introduce an outer cutoff radius to truncate the population so that null results from direct-imaging observations are consistent with the model (e.g., Reggiani et al. 2016; Nielsen \& Close 2010). We note that calibration of the evolutionary models needed to estimate masses from luminosities and temperatures is still underway and represents an important caveat for direct-imaging searches.

Attempts to test whether similar fits can be used to describe exoplanet populations surrounding both higher and lower mass stars have revealed a dependence of either the overall planet occurrence or of the planet mass function on stellar mass: gas giant planets are detected with greater frequency around stars of higher mass (e.g., Johnson et al. 2010) at least up to $3.0 M_{\odot}$ (Reffert et al. 2015). The microlensing technique offers a unique opportunity to assess the exoplanet population at intermediate separations down to very low planet masses, predominantly around low-mass M-dwarf primaries (Gould et al. 2010). However, care must be taken in comparing the microlensing results to those from other techniques. First, we do not accurately know the host star mass of most of these microlensing events (Fukui et al. 2015). Second, it is well known that the bulk system metallicity affects the outcome of gas giant planet formation (e.g., Valenti $\&$ Fischer 2005): the microlensing sample, probing the galactic bulge (3-10 Gyr), may be metal poor compared to younger field star samples probed by RV and direct imaging. Knowing the orbital distribution of exoplanets, even over a limited mass range, is vital to test theories of planet formation and subsequent orbital evolution (cf. Mordasini et al. 2012).

Given the possibility of combining microlensing results with those from other techniques, low-mass stars provide a unique opportunity to study exoplanet surface density distributions. Previous attempts to reconcile surveys of gas giants surrounding $\mathbf{M}$ dwarfs with gas giants of higher mass stars led to the conclusion that either the planet mass function surrounding $\mathrm{M}$ dwarfs must rise sharply to lower mass, or the normalization and/or orbital distribution must be different 
Table 1. Observational constraints on $f$ (number of 1-10 $M_{J}$ planets per star) vs. orbital separation.

\begin{tabular}{lll}
\hline \hline Semimajor axes (AU) & $f_{\text {Cassan }}^{a}$ & Reference \\
\hline $0.07-0.33$ & $0.008_{-0.005}^{+0.0017}$ & Bonfils et al. (2013) \\
$0.5-10.0$ & $0.063 \pm 0.0375$ & Cassan et al. (2012) \\
$0-20$ & $0.0625 \pm 0.03$ & Montet et al. (2014) \\
$10-100$ & $<0.100 \pm 0.001^{b}$ & Bowler et al. (2015) \\
$8-400$ & $0.022_{-0.007}^{+0.028}$ & Lannier et al. (2016) \\
\hline
\end{tabular}

Notes. ${ }^{(a)}$ Assuming the planet mass function of Cassan et al. (2012). ${ }^{(b)}$ For cold-start models, $<0.154 \pm 0.002$.

(Quanz et al. 2012; Clanton \& Gaudi 2014). More recently, Clanton \& Gaudi (2016) have shown that exoplanet statistics from RV (Montet et al. 2014), microlensing (Gould et al. 2010), and direct imaging (Bowler et al. 2015) can be consistently fit using a steep power-law in the surface density distribution that is abruptly truncated at orbital radii smaller than $15 \mathrm{AU}$. Here we take an alternate approach and suggest a different but well-motivated functional form that also fits the most recent observational data.

\section{Why log-normal?}

It is well known that the semimajor axis distribution of stellar binary companions surrounding FGK stars can be fit by a lognormal that peaks at about $50 \mathrm{AU}$ (Raghavan et al. 2010). The surface density of stellar companions surrounding $\mathrm{M}$ dwarfs and A stars also follows a log-normal, with the mean proportional to the host star mass Janson et al. (2012) and De Rosa et al. (2014). While models have been developed based on the idea that significant dynamical evolution in young star clusters is responsible for the observed log-normal period distribution (Marks \& Kroupa 2012), it is possible that the distribution is primordial, reflecting the outcome of the star formation process (Parker \& Meyer 2014; Offner et al. 2010). If stellar (and substellar) companion formation surrounding stars (and any subsequent orbital evolution) results in a log-normal semimajor axis distribution, perhaps planet formation does as well. While gravitational instability probably only occurs in rare circumstances (Vigan et al. 2017), it could give rise to different mass and orbital separation distributions compared to the core accretion model (Santos et al. 2017). We know that gas planet formation via core accretion is complex, resulting from many independent processes (growth of solids, build-up of a core with critical mass, and runaway gas accretion), and the further evolution of their orbital radii depends on many factors (Benz et al. 2014; Helled et al. 2014). In the limit of a product of an infinite number of independent variables, regardless of their underlying distributions, the central limit theorem dictates an outcome in the form of a log-normal. In reality, a limited number of variables rapidly approaches a lognormal (Adams \& Fatuzzo 1996). Thus far, the data for stars over a wide mass range suggest that exoplanet populations exhibit a log-rising frequency at small separations ( $<3 \mathrm{AU}$; Cumming et al. 2008), but they are not common at orbital radii $>30$ AU (Bowler 2016). It seems to us that a log-normal distribution is a reasonable choice of functional form to describe the semimajor axis distribution of exoplanets that appears to rise with logarithmic separation to fall subsequently. Here we demonstrate that the data for M dwarfs are consistent with such a distribution.

\section{Observational constraints on orbital surface density distribution}

Key to our approach is the explicit assumption that the planet mass function does not depend on orbital separation: we are not aware of any data that would require us to reject this null hypothesis (excluding the pileup of hot Jupiters within $0.1 \mathrm{AU}$ ). We also adopt a planet mass function consistent with available data in order to intercompare various surveys. We have adopted constraints on the frequency of exoplanets around M-dwarf primaries over a common range of planetary mass from 1 to $10 M_{J}$ and diverse orbital separations from several different surveys. These results are summarized in Table 1. From the survey of Bonfils et al. (2013), we consider a range of separations from 0.07 to $0.33 \mathrm{AU}$. Bonfils et al. quote a frequency of $0.02_{-0.01}^{+0.03}$ for the average number of planets between 0.3 and $3 M_{J}$ per star based on their data. We adjusted their result to our common mass range assuming the mass function of Cassan et al. (2012) to ensure consistency with the microlensing results discussed below. We also checked that in the range of masses where the survey is complete, the distribution of masses implied by RV observations were consistent with this assumed mass function (based on the available inclination estimates, or assuming the expectation value of $60^{\circ}$ for the inclination). The primary stars in the survey of Bonfils et al. (2013) range from 0.1 to $0.6 M_{\odot}$. These results are consistent with previous other surveys (e.g., Endl et al. 2006) and can also be reconciled with M-dwarf results presented in Cumming et al. (2008) given the uncertainties.

At intermediate separations, we consider results from microlensing surveys that are particularly sensitive to planets in the separation range $0.5-10 \mathrm{AU}$ assuming all hosts are M dwarfs. Adopting the results of Cassan et al. (2012) for the frequency as well as the planet mass function, we estimate the frequency of planets between 1 and $10 M_{J}$ in the above separation range to be $0.063 \pm 0.0375$ planets per star. Although these detections might also include the detection of very low mass brown dwarf companions, such objects are expected to be relatively uncommon given the universal companion mass ratio distribution of Reggiani \& Meyer (2013). This expectation is confirmed in the recent analysis by Shvartzvald et al. (2016), where the companion mass ratio distribution for microlensing events (assuming M-dwarf primaries) shows a local minimum above $10 M_{J}$, consistent with the model for FGK stars in Reggiani et al. (2016).

For the largest orbital separations, we use results from direct-imaging surveys. Here we quote results from Montet et al. (2014), Bowler et al. (2015), and Lannier et al. (2016) regarding the frequency of gas giant planets surrounding primaries between 0.2 and $0.6 M_{\odot}$. Montet et al. (2014) combined results from a long time-baseline RV survey of $111 \mathrm{M}$ dwarfs with direct-imaging constraints that inform the interpretation of accelerations observed without full orbital solutions. They reported a frequency of $0.065 \pm 0.03$ planets per star over the mass range $1-13 M_{J}$ between 0 and $20 \mathrm{AU}$. Bowler et al. (2015) quoted an upper limit of 0.103 planets per star over the range from 10 to $100 \mathrm{AU}$ and 1 to $13 M_{J}$ based on hot-start models of their early evolution: if cold-start models are considered, this limit is recalculated to be 0.16 . Most recently, Lannier et al. (2016) reported a high-contrast imaging survey of $54 \mathrm{M}$ dwarfs, resulting in an estimate for the frequency of $0.023_{-0.007}^{+0.029}$ over a mass range $2-14 M_{J}$ between 8 and $400 \mathrm{AU}$. We adjusted these results assuming the planet mass function from Cassan et al. (2012) for comparison to other surveys over a common mass range of $1-10 M_{J}$ as presented in Table 1. 


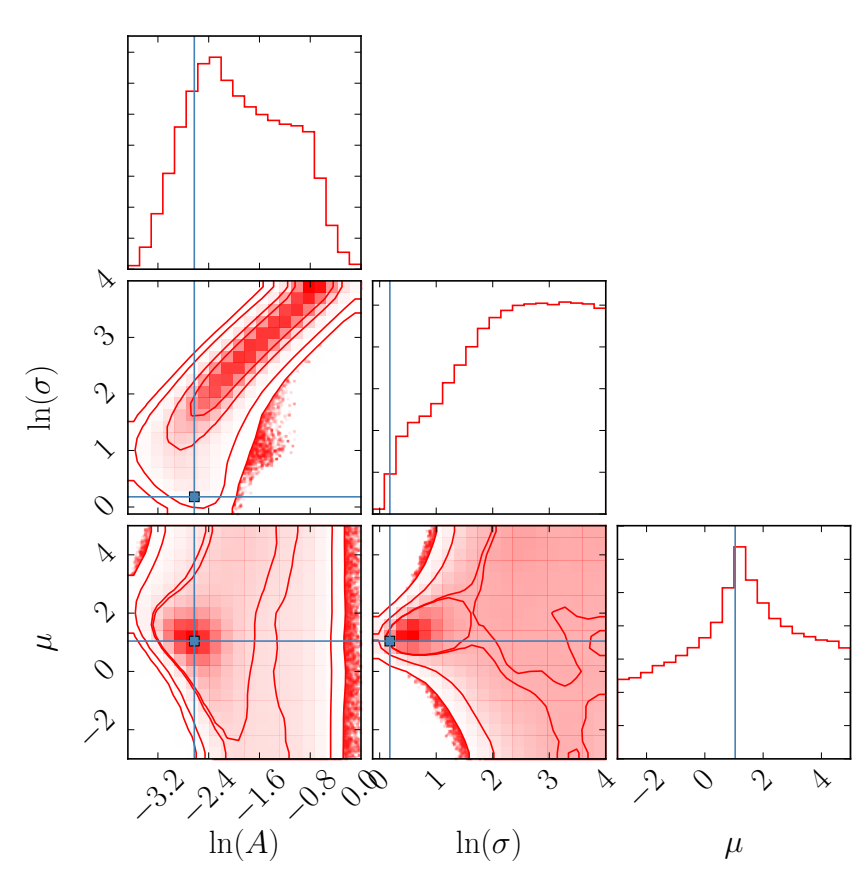

Fig. 1. Probability density functions for the three parameters used in the log-normal fit described in the text: $\ln (A), \ln (\sigma)$, and $\mu$ (red histograms). We also show the correlations between parameters, where reds are highest and whites are lowest values of the likelihoods. Plotted in blue are the locations of the parameter values in the MLE fit described in the text.

\section{Results}

Using constraints from these data (including the upper limit) and our assumption of a log-normal functional form, we explored allowed values of three parameters (amplitude $(A)$, mean of the log-normal $(\mu)$, and square root of the variance $(\sigma)$, all restricted to be positive) using a Markov chain Monte Carlo approach to survey the landscape of the likelihood function with the publicly available package CosmoHammer (Akeret et al. 2013). We adopted a probability density function (hereafter PDF) for the RV planet frequency from Bonfils et al. (2013; as well as the direct-imaging results of Lannier et al. 2016) that accurately reflect the reported asymmetric error bars (a log-normal). For the microlensing results of Cassan et al. (2012) as well as those of Montet et al. (2014) we assumed a Gaussian with a mean and $\sigma$ corresponding to the reported values and uncertainties. We adopted a complementary error function for the hot-start results of Bowler et al. (2015) with the mean as the upper limit and a $\sigma$ of 0.001 . We assumed flat priors in the log for each free parameter, with $-4<\ln (\sigma)<4$. Beyond $\ln (\sigma)>2$, all fits are equal and not as good as fits with $\ln (\sigma)<2$, but they are difficult to formally rule out, which is why we quote the MLE below. We then obtained a PDF for each variable, marginalized over the other two variables, and the correlations between variables as shown in Fig. 1. While the amplitude and mean are reasonably well constrained, the width of the distribution is not. This results in the degeneracy between $\sigma$ and amplitude as well as structure in the $\sigma$ versus $\mu$ plot: when $\sigma$ becomes very large, $\mu$ is poorly constrained and the amplitude is adjusted accordingly. We also calculated the maximum likelihood estimate (MLE) that maximizes the chance of these data being drawn from our model, the parameter values of which are also denoted in Fig. 1. Here, $\phi(x)$ is the PDF of having a gas giant planet $1-10 M_{J}$ as a function

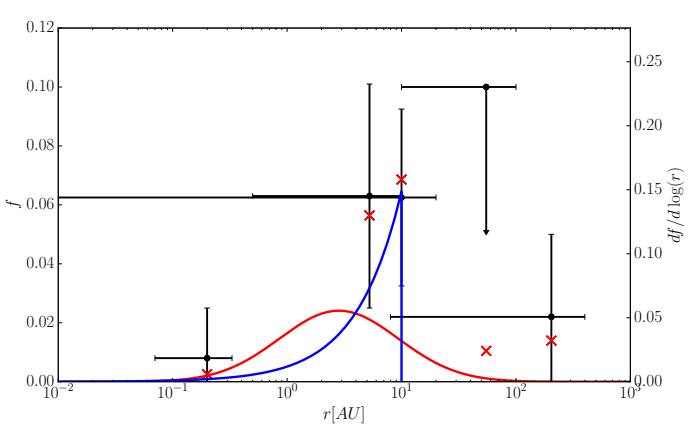

Fig. 2. Log-normal surface density distribution of 1-10 $M_{J}$ companions to $\mathrm{M}$ dwarfs. On the left axis we plot the integrated values of $\mathrm{f}$, where the black points correspond to the data points listed in Table 1: note the horizontal lines are ranges, while the vertical lines are errors in $\mathrm{f}$. On the right axis, we show the relative values of the differential PDFs. In red, we show the MLE fit as described in the text. The integrated $f$ values for the MLE, over the ranges in semimajor axis corresponding to the data from Table 1, are shown as a red cross. We show in blue the fit from Clanton \& Gaudi (2016) that was published before the results of Lannier et al. (2016) were available.

of the orbital semimajor axis $x$, and $f$ is the frequency over the limits of integration:

$\phi(x)=\frac{\mathrm{d} f}{\mathrm{~d} x}=A \frac{e^{(\ln (x)-\mu)^{2} / 2 \sigma^{2}}}{x 2 \pi \sigma}$.

The MLE has parameters $\ln (A)=-2.6267, \ln (\sigma)=0.1801$, $\mu=1.0413$. In base ten $\phi(x)=A \frac{e^{(\log (x)-\mu)^{2} / 2 \sigma^{2}}}{2 \pi \sigma}$ with same amplitude $A, \mu=0.4527$, and $\sigma=0.5205$. We present this fit (in red) in Fig. 2 together with the integrated frequencies over the ranges indicated from our fits for comparison with the data in Table 1. This distribution, with a mode at $2.8 \mathrm{AU}$, yields 0.07 planets per star over the mass range from 1 to $10 M_{J}$ over all separations (assuming the planet mass function from Cassan et al. 2012), and it satisfies the observed constraints.

\section{Discussion}

In the MLE, half of all planets with masses $1-10 M_{J}$ are within $2.8 \mathrm{AU}$ and half are beyond, with a prediction of $0.015<f<$ 0.03 (at $80 \%$ confidence) from 10 to 1000 AU. Clanton \& Gaudi (2016) recently fit similar data (excluding the new result of Lannier et al. 2016) with a continuous power-law that rose sharply to account for the microlensing detections, but then abruptly truncated the planet population as a result of the directimaging upper limits from Bowler et al. (2015) (shown in blue in Fig. 2). This implied that no gas giant planets exist beyond $10 \mathrm{AU}$. We prefer the log-normal to model this rise and fall of the orbital surface density distribution of gas giant exoplanets, which is also consistent with the new estimates of Lannier et al. (2016), which indicate that a small but non-zero fraction of gas giant planets lie beyond $10 \mathrm{AU}$.

Additional observations that would better constrain the width of the log-normal are crucial. For example, next-generation microlensing surveys could significantly improve upon the precision of the estimated frequency of exoplanets between 0.5 and $10 \mathrm{AU}$ (e.g., Gaudi 2012). Future high-contrast imaging surveys with ground- and space-based telescopes will help place important constraints on $\sigma$. For example, a survey with the NIRCam instrument on the JWST will be able to measure $f$ much more precisely, down to much lower masses, from 10 
to $100 \mathrm{AU}$ around very nearby young M dwarfs (e.g., Schlieder et al. 2016). In the next decade, the ELTs will be able to image planets across this expected peak in the log-surface density distribution at about $3 \mathrm{AU}$. Does the location of the peak in the log-normal surface density distribution depend on stellar mass? If it is related to photoevaporation (as a stopping point in the inner migration of gas giant planets formed at large separations), then perhaps the answer is yes (e.g., Alexander \& Pascucci 2012; Ercolano \& Rosotti 2015) because lower mass stars are expected to have peaks closer to their stars. However, phenomenological predictions of core accretion, relating the time required to reach a critical core mass for runaway gas accretion as a competition between disk lifetime (which depends inversely on stellar mass) and the rate of collisions (which is faster around higher mass stars with more massive disks) suggest a weak dependence. On the other hand, if the water-ice line is critical to gas giant planet formation, we expect a linear dependence of this quantity on stellar mass in the pre-main sequence where $L \sim M^{2}$ (cf. Kennedy $\&$ Kenyon 2008). When we assume that the overall frequency of gas giant planet formation depends linearly on stellar mass, our model predicts $f=0.06$ for the frequency of planets 1$10 M_{J}$ between 10 and $100 \mathrm{AU}$ around solar-mass stars, or even higher if the mode of the log-normal depends linearly on stellar mass. So far, these numbers are consistent with estimates for the frequency of gas giants on wide orbits from legacy RV surveys (see Wittenmyer et al. 2016; Durkan et al. 2016), but this will be put to a strong test by ongoing FGK star surveys such as are being carried out now with SPHERE and GPI. Extrapolating the amplitude of our fitted function to A stars with a linear mass dependence, but not adjusting the mode, we would expect $f=0.12$ from 1 to $10 M_{J}$ from 10 to $100 \mathrm{AU}$ surrounding A star samples, consistent with current constraints (e.g., Vigan et al. 2012). In a future paper, we will explore the stellar mass dependence of exoplanet populations concretely, with a focus on the planet mass function.

Acknowledgements. We thank an anonymous referee for a helpful review, as well as other anonymous referees, who commented on earlier versions of this manuscript. This work has been carried out in part within the frame of the National Centre for Competence in Research PlanetS supported by the Swiss National Science Foundation. MRM and SPQ are pleased to acknowledge this financial support of the SNSF. M.R. acknowledges funding from the European Research Council Under the European Union's Seventh Framework Program (ERC Grant Agreement No. 337569) and from the French Community of Belgium through an ARC grant for Concerted Research Action.

\section{References}

Adams, F. C., \& Fatuzzo, M. 1996, ApJ, 464, 256

Akeret, J., Seehars, S., Amara, A., Refregier, A., \& Csillaghy, A. 2013, Astron. Comput., 2, 27
Alexander, R. D., \& Pascucci, I. 2012, MNRAS, 422, L82

Benz, W., Ida, S., Alibert, Y., Lin, D., \& Mordasini, C. 2014, Protostars and Planets VI, 691

Beuzit, J.-L., Feldt, M., Dohlen, K., et al. 2008, in Ground-based and Airborne Instrumentation for Astronomy II, Proc. SPIE, 7014, 701418

Bonfils, X., Delfosse, X., Udry, S., et al. 2013, A\&A, 549, A109

Bowler, B. P. 2016, PASP, 128, 102001

Bowler, B. P., Liu, M. C., Shkolnik, E. L., \& Tamura, M. 2015, ApJS, 216, 7

Cassan, A., Kubas, D., Beaulieu, J.-P., et al. 2012, Nature, 481, 167

Chauvin, G., Vigan, A., Bonnefoy, M., et al. 2015, A\&A, 573, A127

Clanton, C., \& Gaudi, B. S. 2014, ApJ, 791, 91

Clanton, C., \& Gaudi, B. S. 2016, ApJ, 819, 125

Cumming, A., Butler, R. P., Marcy, G. W., et al. 2008, PASP, 120, 531

De Rosa, R. J., Patience, J., Wilson, P. A., et al. 2014, MNRAS, 437, 1216

Durkan, S., Janson, M., \& Carson, J. C. 2016, ApJ, 824, 58

Endl, M., Cochran, W. D., Kürster, M., et al. 2006, ApJ, 649, 436

Ercolano, B., \& Rosotti, G. 2015, MNRAS, 450, 3008

Fischer, D. A., \& Valenti, J. 2005, ApJ, 622, 1102

Fukui, A., Gould, A., Sumi, T., et al. 2015, ApJ, 809, 74

Gaudi, B. S. 2012, ARA\&A, 50, 411

Gould, A., Dong, S., Gaudi, B. S., et al. 2010, ApJ, 720, 1073

Heinze, A. N., Hinz, P. M., Kenworthy, M., et al. 2010, ApJ, 714, 1570

Helled, R., Bodenheimer, P., Podolak, M., et al. 2014, Protostars and Planets VI, 643

Janson, M., Hormuth, F., Bergfors, C., et al. 2012, ApJ, 754, 44

Johnson, J. A., Aller, K. M., Howard, A. W., \& Crepp, J. R. 2010, PASP, 122 905

Kennedy, G. M., \& Kenyon, S. J. 2008, ApJ, 673, 502

Lafrenière, D., Doyon, R., Marois, C., et al. 2007, ApJ, 670, 1367

Lannier, J., Delorme, P., Lagrange, A. M., et al. 2016, A\&A, 596, A83

Macintosh, B., Graham, J. R., Ingraham, P., et al. 2014, Proc. Natl. Acad. Sci., 111,12661

Marks, M., \& Kroupa, P. 2012, A\&A, 543, A8

Mayor, M., Marmier, M., Lovis, C., et al. 2011, ArXiv e-prints [arXiv: 1109.2497]

Montet, B. T., Crepp, J. R., Johnson, J. A., Howard, A. W., \& Marcy, G. W. 2014, ApJ, 781, 28

Mordasini, C., Alibert, Y., Klahr, H., \& Henning, T. 2012, A\&A, 547, A111

Nielsen, E. L., \& Close, L. M. 2010, ApJ, 717, 878

Offner, S. S. R., Kratter, K. M., Matzner, C. D., Krumholz, M. R., \& Klein, R. I. 2010, ApJ, 725, 1485

Parker, R. J., \& Meyer, M. R. 2014, MNRAS, 442, 3722

Quanz, S. P., Lafrenière, D., Meyer, M. R., Reggiani, M. M., \& Buenzli, E. 2012, A\&A, 541, A133

Raghavan, D., McAlister, H. A., Henry, T. J., et al. 2010, ApJS, 190, 1

Reffert, S., Bergmann, C., Quirrenbach, A., Trifonov, T., \& Künstler, A. 2015, A\&A, 574, A116

Reggiani, M., \& Meyer, M. R. 2013, A\&A, 553, A124

Reggiani, M., Meyer, M. R., Chauvin, G., et al. 2016, A\&A, 586, A147

Santos, N. C., Adibekyan, V., Figueira, P., et al. 2017, A\&A, 603, A30

Schlieder, J. E., Beichman, C. A., Meyer, M. R., \& Greene, T. 2016, in Young Stars Planets Near the Sun, eds. J. H. Kastner, B. Stelzer, \& S. A. Metchev, 288

Shvartzvald, Y., Maoz, D., Udalski, A., et al. 2016, MNRAS, 457, 4089

Valenti, J. A., \& Fischer, D. A. 2005, ApJS, 159, 141

Vigan, A., Patience, J., Marois, C., et al. 2012, A\&A, 544, A9

Vigan, A., Bonavita, M., Biller, B., et al. 2017, A\&A, 603, A3

Wittenmyer, R. A., Butler, R. P., Tinney, C. G., et al. 2016, ApJ, 819, 28 